\title{
Applying New Educational Models in ICT Teaching and Learning
}

\author{
Evandro Cantú ${ }^{1}$ and Jean Marie Farines ${ }^{2}$ \\ 1 Federal Center of Technological Education of Santa Catarina (CEFET- \\ SC) São José, SC, Brazil cantu@sj.cefetsc.edu.br, \\ WWW home page: http://www. sj.cefetsc.edu.br/ cantu \\ 2 Department of Automation and System (DAS), Federal University of \\ Santa Catarina (UFSC) Florianópolis, SC, Brazil farines@das.ufsc.br, \\ WWW home page: http://www. das.ufsc.br/ farines
}

\begin{abstract}
This paper presents a group of elements to improve the teaching and learning in technological education, applied to Information and Communication Technologies (ICT). These elements are based on some contributions issued from contemporary education models, namely, the thematic approach, meaningful learning, concept maps and the spiral curriculum. Based on a thematic approach, a new way is proposed to select the contents that will be explored in the educational process. We emphasize the benefits of a thematic approach in students' motivation. After contents are chosen, we propose the use of the directives of meaningful learning and concept maps, as a method and a tool, respectively, to organize knowledge hierarchically, from general and inclusive concepts to the specific ones. This may facilitate the learning and understanding of the great bulk of knowledge to be covered in technological courses. A proposal has been submitted for a teaching module in ICT. The spiral curriculum was applied during the development of learning, where some fundamental concepts were introduced many times over, increasing more details gradually. A qualitative analysis of the application of these educational proposals, including learning evaluation aspects, is also presented.
\end{abstract}

\section{Introduction}

In technological courses the curricula are generally organized by following the classical structure of the programmatic contents of a domain, using a textbook as the main didactic material. 
The rigid use of textbook material and its high complexity and great diversity of content does not motivate students, as it imposes great difficulties on them in the understanding of concepts and how to relate the topics being studied with real applications.

Furthermore, in areas with a continuous change of technological content, as with information and communication technologies (ICT), another problem is the difficulty in selecting and organizing the knowledge to be taught. On the one hand, new knowledge has to be added to the curriculum constantly, and at the same time any other knowledge becomes obsolete. On the other hand, content has to be organized and ordered, relating every concept to others, which is not a trivial task because of their number and how often they change.

With the aim of dealing with these questions, this paper presents a group of elements to improve teaching and learning in ICT education. These elements are based on modern educational models, namely, the thematic approach, meaningful learning, concept maps and the spiral curriculum. The second section presents the methodological basis of these educational contributions. The third section presents a set of guidelines to select and organize the content of technological courses. Based on these guidelines, a proposal has been submitted for a teaching module in ICT, which is presented and discussed in the fourth section. Finally, the conclusion presents a qualitative analysis of the application of this educational proposal.

\section{Methodological Considerations}

This section presents educational models used as elements to improve the teaching and learning of technological education. The models considered in this work are the thematic approach, meaningful learning, concept maps and the spiral curriculum.

\subsection{Thematic approach}

The thematic approach was introduced by Freire $[1]^{2}$ and made some important contributions to the educational process. Although working with alphabetization, his ideas have been applied to general education, including also educational science [2]. According to Freire, learning activities must be developed around generative themes that are part of the students' cultural environment. These generative themes increase the students' motivation and allow them to extend their knowledge about the subject, including social and political factors, which can contribute to the production of complete professionals and citizens with innovative and critical minds. This work is positioned in this context.

According to [2], the attraction of the new technological systems and the discussion of the relationship between technology and society are two important requirements in the selection of the generative themes. For these authors, the generative themes present a "rupture" in the way the curricula have been elaborated, which are, normally, strongly based on content and organized in a rigid and

${ }^{2}$ Most books of Freire have been translated into English; see www. paul of reire. org. 
systematic way. In a dynamic domain, with a lot of changing content, as in technological courses on ICT, the use of generative themes is a good criterion for helping in the selection of content.

In a domain related to ICT, the generative themes can be issued from applications that people commonly use, like the Internet or mobile systems. For example, to teach computer networks, some Internet applications present in home or working situations match the requirements to be selected as generative themes.

Mobile systems and the Internet, their relationship and social consequences, have to be considered as starting points in order to learn about telecommunications and digital convergence, since a new way of life is emerging in all countries, with social inclusion as an important requirement, mainly in developing countries.

\subsection{Meaningful learning and concept maps}

The concept of meaningful learning was first presented by Ausubel [3] and can be considered as a pedagogical theory for classroom learning, which explains how the students learn and understand the formal knowledge that is received in school [4].

For this model, the learning occurs when new information is obtained from a planned effort by the learner to link this information with some relevant concepts already existing in his/her cognitive structure. To accomplish this task, the suggestion is to start learning from more general and inclusive concepts and then to go towards the more specific ones.

When translating this kind of learning into practical terms, one important task is to determine the conceptual structure of the domain to be taught. In other words, we have to identify the underlying concepts and principles of the domain and organize them hierarchically. Afterwards, this conceptual structure can be used to lend the learning an optimal sequence.

Concept maps, developed by Novak [5-6] in a sequence of research on meaningful learning, help in this task. Concept maps are a kind of graph used to represent the relationships among a group of concepts. A concept can be associated with each node of the graph. The labeled links between nodes show the relationships among concepts. The links are read from top to bottom, or, if there are arrows, following them. Figure 2 is a simple example of a concept map describing a computer network.

In this representation concepts are organized in a hierarchical way, with the most general and inclusive concepts at the top and the more specific ones below. However, for a concept corresponding to a node, it is also possible to build another concept map with the objective of refining it (in section 4, Figures 2 and 3 exemplify this situation). Other characteristics of a concept map are the cross-links that interconnect concepts in different regions of the map. For example, in Figure 1 there is a cross-link relating the concepts "Host" and "Network Applications". Examples can also be included in the map in order to clarify the meaning of a specific concept, as in the examples of "Network Applications" (FTP, WWW, Email) in the same figure. 


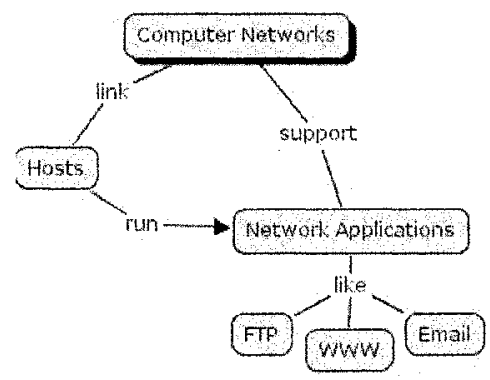

Figure 1. A simple concept map describing a computer network.

To construct "good" concept maps, Novak proposes some directives, described in [5]. Computational tools, like CmapTools [7] $]^{3}$, which we used in our work, also enable the construction of concept maps.

The use of concept maps has increased in recent years, in particular in educational activities ${ }^{4}$. One of the main uses is in curriculum planning. In this activity the concept maps help clearly build and present the content organization and relationships between different parts of the course.

Concept maps can also be used during the development of learning in the classroom. They can be used, for example, as a knowledge organizer to introduce a topic or to synthesize a lecture by integrating different concepts discussed earlier. Sometimes, they are also used as learning and evaluation tools, with the students constructing their own maps of the topic they are studying.

In any discipline related to ICT there are many complex concepts and technologies linked in an intricate way. So, tools like concept maps can be of great assistance in organizing and structuring the knowledge. They can identify the general concepts prior to instruction and assist in the sequencing of learning in a progressive way, from general to more specific and explicit knowledge.

\subsection{Spiral curriculum}

From the same epistemological foundations of meaningful learning, Bruner [8] concluded that learners acquire new ideas or concepts mainly from their past knowledge.

For this educator, a way to guarantee that the present learning will be useful in the future is learning the base structure of the topic in hand. Getting the base structure consists in understanding the stable and fundamental concepts which underline the topic and that can be related to many other things or situations.

Bruner proposes, during the learning process, to return to the fundamental concepts many times when following a spiral curriculum. The idea is to explore the concepts repeatedly, increasing each time their depth and detail.

3 Cmap Tool is a tool to construct concept maps of the Institute for Human and Machine Cognition, USA. http: / / cmap. ihmc . us

${ }^{4}$ As we can see in the Proceeding of the First International Conference on Concept Mapping

(CMC2004), Pamplona, Spain. http: / / cme. ihme.us 
In a dynamic and changing domain such as ICT, our proposition is to use the spiral curriculum during the development of learning, to assure a full learning of fundamental and lasting concepts. These concepts are important to understand, for example, not just current but future technologies as well.

\section{Guidelines to select and organize content in technological courses}

From the educational models presented in the last section, we recommend some guidelines to help in the selection and organization of content in technological courses.

We propose three steps to be followed by teachers, after the scope of the course has been defined:

1. Set the bounds of knowledge to be taught by using a thematic approach;

2. Identify the most fundamental and lasting concepts;

3. Organize hierarchically the knowledge according to the meaningful learning model and modeling it by using concept maps.

To set the bounds of knowledge we propose the use of generative themes, as the thematic approach suggests. The domain to be taught defines the context in which to identify and choose the generative themes. These are tools or applications normally used by the students, which can be technological products of the domain. For example, as mentioned in subsection 2.1 , the Internet and mobile systems can be chosen as a generative theme in the ICT domain.

The generative theme is used as a vehicle to teach the underlying concepts of the domain to be studied. The first benefit of this approach is to increase the students' motivation, working with material and applications used by and familiar to the students. Another benefit is the possibility of extending the discussion to subject matter relating to the dynamic between technology and society, increasing the students' general knowledge and preparing them for professional life.

After choosing the generative theme, the second step of our proposal requires that the teacher determine the scientific and technological knowledge essential to the understanding of the theme. This time, all the fundamental and lasting concepts must be carefully defined. It is important to observe that not all the contents normally considered in a traditional approach are taken into account when we use a thematic approach. The thematic approach makes a kind of cut in the group of contents, keeping those related to the theme chosen. Regarding this cut, we consider the full understanding of fundamental concepts more important than studying all the traditional contents extensively. A student who understands a real system or technology well, including all its fundamental concepts and also the aspects related to the impact of this technology in society, will be well prepared to understand any new technology in the future.

The next step is to organize the knowledge hierarchically, by using the directives of meaningful learning. For this particular context, the fundamental concepts could be listed, and then, from this list a rank order could be established from general and 
inclusive concepts to the more specific ones, allowing the building of a kind of hierarchical tree.

Finally, the modeling of this knowledge can be made by using concept maps. The arrangement constructed previously is helpful in starting the modeling. Now, each particular concept must be related to its parent using a word or a sentence that characterizes the relation. Concepts in different parts of the map under construction can also be interrelated, forming cross-links. As almost all concepts could be related in different ways, we must be judicious in choosing the meaningful cross-links we desire. During the construction of the model, we can also refine a concept in a new map, forming a three-dimensional hierarchical structure.

The resulting knowledge model, formed by the collection of concept maps, can be used for teaching planning, establishing an optimal sequence to develop the content.

To complement these guidelines we also propose the application of spiral curriculum during the development of learning in the classroom. The recommendation is to explore the fundamental concepts many times over, going deeper and detailing each time.

The next section describes an application example of these guidelines for a teaching module in the ICT domain.

\section{An ICT Application: a teaching module on computer networks}

This section describes an application example of our proposal, which was implemented in a teaching module on computer networks in the Federal Center of Technological Education of Santa Catarina, in Brazil.

\subsection{Building a knowledge model for computer networks}

The pre-defined scope of the considered teaching module consists of a general computer networks course for industrial engineers, including topics such as network services, protocols, architecture and applications.

Different from classical computer networks courses, our thematic approach used the Internet applications as a starting point in order to structure the course. All the technical contents of the teaching module were directly obtained from these generative themes. The objective was the construction of a structural vision of the Internet, which would include the Internet protocol architecture and its related technologies such as accessing networks, local area networks and wireless networks.

Figure 2 illustrates a concept map built from the chosen generative themes. In this figure, the labeled link "use vision" points towards classical Internet applications, the link "structural vision" towards layers of Internet architecture. The link "access networks" points towards local area networks and technologies for "last mile" access. Some cross-links were also included in the concept map, showing the relationship between concepts, in particular, relating specific concepts and technologies with the "layers" of Internet architecture. 


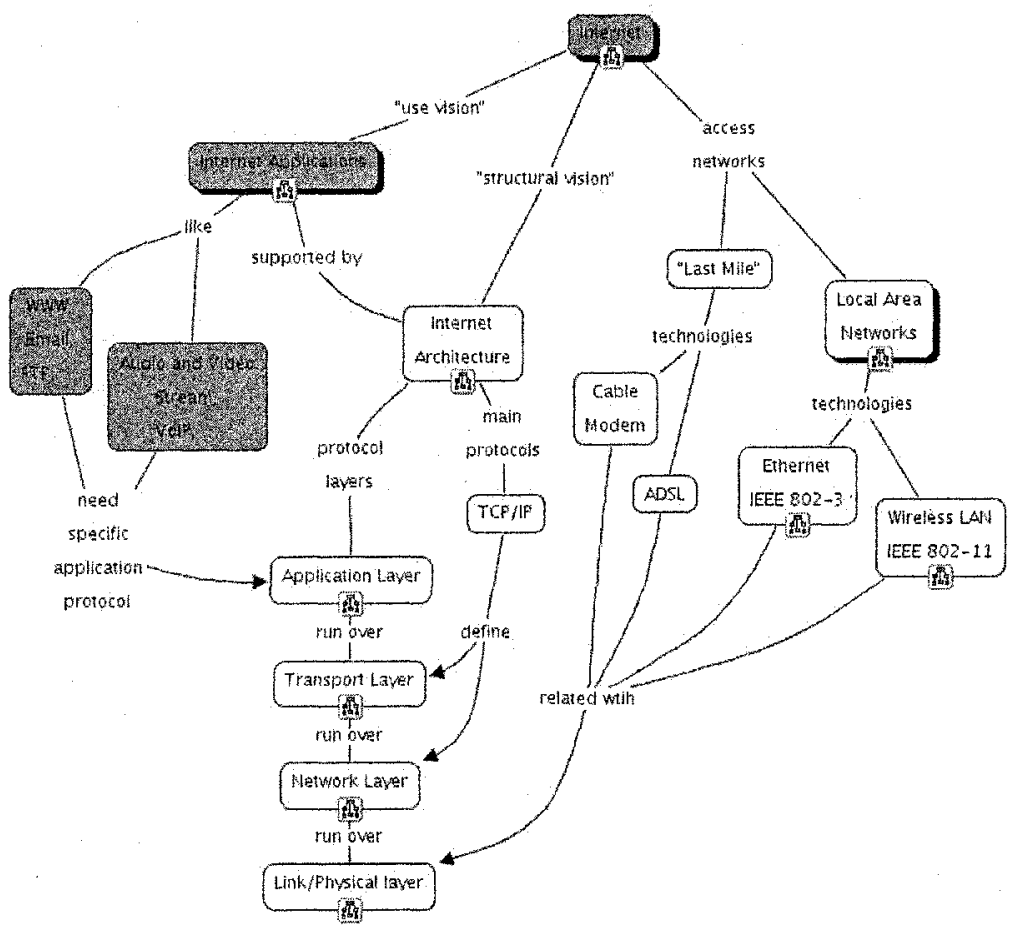

Figure 2. Concept map built from generative themes about Internet.

In the concept map representation, the icons under a concept mean that another map details this concept. As represented in Figure 2, the main concepts and technologies related with the Internet were refined and modelled with other concept maps. For example, Figure 3 details part of Figure 2, corresponding to the organization of the "Transport Layer" of the Internet architecture.

To construct this knowledge representation we considered the classical knowledge of computer networks that supports the Internet and then we organized the concepts from a general to specific way. The complete knowledge model used during the development of this teaching module makes part of a Web environment ${ }^{5}$, presented in [9].

\subsection{Development of the teaching module in the classroom}

During the development of this teaching module in the classroom, two educational activities were included in the course, aiming to introduce the generative themes. The first activity was related to a "use vision" of the Internet applications. The objective was to motivate the students and show how useful the network applications were in improving their daily task management. Some laboratory activities were carried out, involving the use of applications like WWW, FTP, Email and remote 
login. The second activity was related to the impact of the Internet on contemporary society, researched by the students on the web, and the organization of a classroom debate.

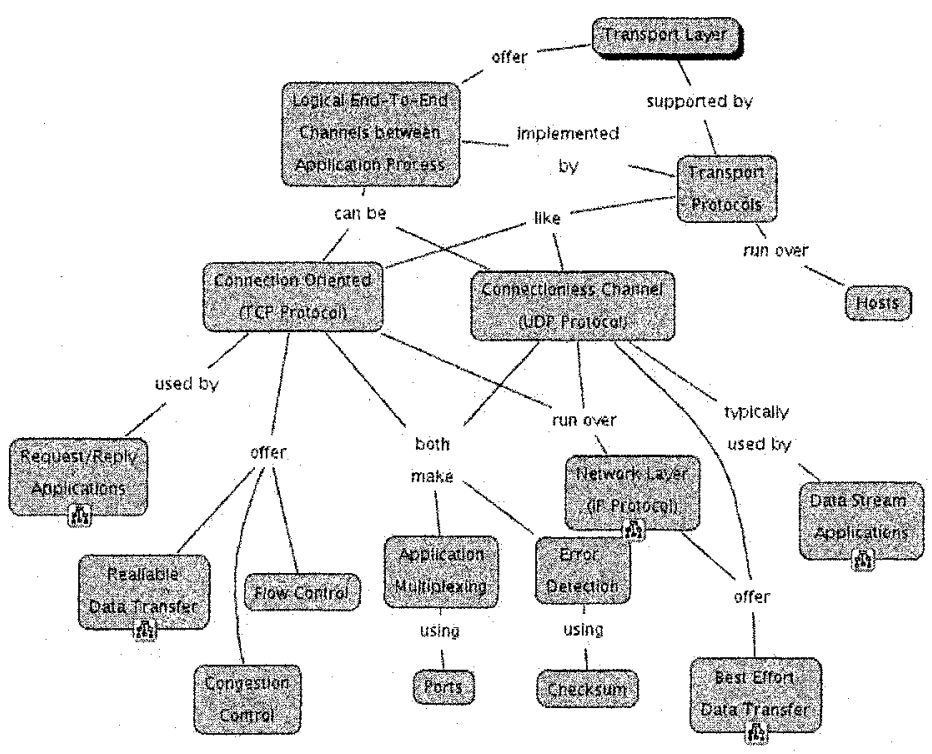

Figure 3. Internet "Transport Layer" organization.

After these introductory activities, we used the previously built concept maps representing the knowledge of the Internet in the classroom in different manners: firstly, as an advanced tool to show the content organization and the relationship between different parts of the course; secondly, before the development of a specific topic to show the different concepts involved in the discussion; and thirdly, after the lectures to make a synthesis of the discussed concepts. By putting these activities in practice, the teacher provided and discussed the concept maps and the related content with the students.

The concept maps helped the teacher to orient his students to use Web materials and technical reports associated with network technologies. For example, when we discussed the "last mile" technologies (ADSL and cable modem), we used the existing concept maps and we requested that students carry out a Web search to complete them. We also furnished them with some materials, such as technical manuals of modems and equipments to be tested. The students worked in groups and we required that each group construct a concept map representation of the topics. Each group of students constructed its own concept maps and we evaluated these maps in a feedback instruction. In this case, we emphasize that the activity of constructing concept maps is a powerful learning tool.

The concept maps were also used as an evaluation tool, complemented by traditional evaluation activities. The concept maps built by students allow them to evaluate the understanding of each one and to get individual feedback. We verified 
that concept maps are very effective in identifying both valid and invalid thoughts held by students, improving the students' continuous evaluation and the feedback.

Although featuring the Internet, we emphasized the fundamental and lasting concepts of general computer networks, which allow the students to understand any other network technology. To consolidate the understanding of these fundamental concepts, we used the spiral curriculum model, presented in subsection 2.3. During the learning process, the main concepts of "packet switching", "protocol layers", and others, were developed many times, exploring them in more detail every time. For example, for the concept of "reliable data transfer", a very important concept used in packed switched networks, we first discussed the topic and its general meaning by using analogies (we used an analogy based on a postal purchase of an encyclopedia made of fascicles). After that, during the presentation of the Internet protocol TCP, we used some simple exchange message diagrams to illustrate how the protocol works. Finally, we dealt with the details of the TCP protocol, its reliability and congestion mechanisms, returning again and again to the fundamental concepts to be learned. The set of concept maps modelling the knowledge of computer networks helped us to reach the learning objectives and realize these activities.

\section{Conclusion}

This paper presented a group of elements to improve teaching and learning in ICT education, which is extensively detailed in [10]. Some modern educational models were applied. A thematic approach was used to select the contents to be taught, increasing the students' motivation. Meaningful learning and concept maps were used as a method and a tool, respectively, to organize knowledge hierarchically, facilitating the learning of the technological content. The spiral curriculum approach was applied to consolidate the understanding of fundamental concepts of the domain being studied. A set of guidelines to select and organize content in technological courses were proposed. An ICT application using our proposal was also presented and discussed.

We emphasize the benefits of concept maps to hierarchically structure the knowledge to be taught, explicitly showing how every concept is related to each other to make the whole, in opposition to the normal organization of textbooks with topics and subtopics, which does not facilitate the development of concepts in a general to specific way. Reinforcing the proposal of [7], concept maps and related "computational tools" allow a new educational model, putting into practice in a simple manner some pedagogical ideas that before presented difficulties.

Teachers can use these pedagogical elements in different situations. 1) To plan a course, applying the guidelines proposed in section 3 to select and organize content in technological courses. In the ICT domain, teachers can use the existing concept maps about computer networks, available on the Web, as a knowledge organizer to plan a course, or using computational tools such as CmapTools [7], teachers can also make modifications or construct new concept maps, following the meaningful learning model. 2) During the development of a course in the classroom, teachers can use concept maps as shown in section 4, eventually using generative themes or 
other examples to motivate the students, and apply the principles of spiral curriculum to consolidate the learning of fundamental and lasting concepts. 3) Teachers can also use concept maps as an evaluation tool, giving a continuous feedback on the learning process.

However, the success of any educational proposal depends on the teachers' initiative. There are no ready-made prescriptions. What we intend to do with this work is not to impose a method, but to offer teachers an aid to improve their pedagogical activities.

The environment presented in [9], with a previous set of concept maps about computer networks, is available on the Web, including Portuguese and English versions. We are also working to enrich this Web environment with other knowledge models and pedagogical materials.

\section{References}

1. Paulo Freire. Educação como Prática da Liberdade (Paz e Terra, Rio de Janeiro,1986).

2. Demétrio Delizoicov, José André Angotti, and Marta Maria Pernanbuco. Ensino de Ciências: Fundamentos e Métodos. Docência em Formação (Cortez, São Paulo, 2003).

3. David P. Ausubel, J. D. Novak, and H. Hanesian. Educational psychology: A cognitive view (Holt, Rinehart, and Winston, New York, 1978).

4. Maria Luz Rodrígues Palmero. La teoría del aprendizaje significativo. In Proceedings of the First International Conference on Concept Mapping, volume 1, pages 535-544, Pamplona, Spain (September 2004).

5. Joseph D. Novak. The theory underlying concept maps and how to construct them. Institute for Human and Machine Cognition (USA, 2003). http: //cmap.coginst. uwf.edu/info/printer.html.

6. Joseph D. Novak. A science education research program that led to the development of the concept map tool and a new model for education. In Proceedings of the First International Conference on Concept Mapping, volume 1, pages 457-467, Pamplona, Spain (September 2004).

7. Joseph D. Novak and Alberto J. Cañas. Building on new constructivist ideas and cmaptools to create a new model for education. In Proceedings of the First International Conference on Concept Mapping, volume 1, pages 469-476, Pamplona, Spain (September 2004).

8. Jerome S. Bruner. The Process of Education (Harvard University Press, Cambridge, $1960)$.

9. Evandro Cantú, Jean Marie Farines, and José André Angotti. Using learning theories for education on the web: A web application for teaching and learning computer networks. In Technology Enhanced Learning (TEL04), Proceedings of 18th IFIP World Computer Conference (WCC2004), Toulouse (France, 2004).

10. Evandro Cantú. Elementos para o Fortalecimento da Mediação Docente na Educação Tecnológica: Aplicação no Ensino-Aprendizagem de Redes de Computadores. Tese de Doutorado, Pós-Graduação em Engenharia Elétrica, Universidade Federal de Santa Catarina, (Agosto 2005). 